\title{
EVIDENCIAS QUIMMICAS DE DETERIORO AMBIENTAL EN MANIFESTACIONES RUPESTRES: UN CASO DE ESTUDIO DEL OESTE TINOGASTEÑO (CATAMARCA, ARGENTINA)
} CHEMICAL EVIDENCE OF ENVIRONMENTAL DETERIORATION IN ROCK ART:
A CASE STUDY IN WESTERN TINOGASTA (CATAMARCA, ARGENTINA)

\author{
EUGENIA TOMASINI*, MARA BASILE** \\ NORMA RATTO *** \& MARTA MAIER ****
}

Desde el punto de vista de los estudios químicos y espectroscópicos se discuten los diferentes pasos del trabajo de caracterización analítica de las muestras, focalizándose en los procesos fisicoquímicos que ocurren en las manifestaciones rupestres expuestas a factores ambientales. Se destaca el uso de técnicas vibracionales, tales como la microscopia Raman y la espectroscopia infrarroja, para el análisis de materiales orgánicos e inorgánicos. Se presentan como ejemplo los resultados del análisis realizado sobre las muestras de las imágenes pintadas en dos de los paneles de la cueva de La Salamanca (Depto. Tinogasta, Catamarca) explicitando las evidencias de deterioro mediante la realización de análisis fisicoquímicos.

Palabras clave: deterioro, microscopia Raman, manifestaciones rupestres, hematita, yeso, weddellita, Catamarca

From the chemical and spectroscopic studies perspective, the article discusses the different steps taken to analyze the samples, focusing on the physical-chemical processes occurring in rock art exposed to environmental factors. The study emphasizes the use of vibrational techniques such as Raman microscopy and infrared spectroscopy in the analysis of organic and inorganic materials. As an example, the results of our analyses of samples taken from images painted on two panels in La Salamanca cave (Tinogasta Department, Catamarca) are presented and related to the paintings' deterioration.

Key words: deterioration, Raman microscopy, rock art, hematite, gypsum, weddellite, Catamarca

\section{INTRODUCCIÓN}

A partir de estudios químicos y espectroscópicos puede determinarse la composición de pigmentos y otros materiales utilizados en la realización de las manifestaciones rupestres, contribuyendo así a la definición de los instrumentos y las técnicas de ejecución (Scott \& Hyder 1993; Guineau et al. 2001; Chalmin et al. 2003; Garate et al. 2004; Vignaud et al. 2006). Una investigación arqueológica suele estar orientada a la determinación, por un lado, de la dimensión práctica vinculada con las formas de resolución de las imágenes, contemplando el origen y la preparación tanto del soporte, como de los pigmentos y su aplicación; por otro lado, de la dimensión temporal de su ejecución. En este marco es que surge la necesidad del análisis y la caracterización fisicoquímica de ciertos materiales para contribuir así a la definición de estas dos dimensiones que articulan las interrogantes arqueológicas. En esta línea se encuentra una diversidad de trabajos que han avanzado en la aplicación de distintas técnicas analíticas para profundizar el conocimiento sobre pigmentos y la tecnología utilizada para su preparación y aplicación en la realización de este tipo de manifestaciones visuales (Aschero

* Eugenia Tomasini, Umymfor-Conicet. Departamento de Química Orgánica, Facultad de Ciencias Exactas y Naturales, Universidad de Buenos Aires, Pabellón 2, Ciudad Universitaria, (1428) Ciudad Autónoma de Buenos Aires, Argentina, email: eugeniatomasini@gmail.com

* Mara Basile, Conicet. Museo Etnográfico Juan B. Ambrosetti, Facultad de Filosofía y Letras, Universidad de Buenos Aires, Moreno 350 (1091) Ciudad Autónoma de Buenos Aires, Argentina, email: mara_basile@yahoo.com.ar

* Norma Ratto, Museo Etnográfico Juan B. Ambrosetti, Facultad de Filosofía y Letras, Universidad de Buenos Aires, Moreno 350 (1091) Ciudad Autónoma de Buenos Aires, Argentina, email: nratto@filo.uba.ar

*** Marta Maier, Umymfor-Conicet. Departamento de Química Orgánica, Facultad de Ciencias Exactas y Naturales, Universidad de Buenos Aires, Pabellón 2, Ciudad Universitaria, (1428) Ciudad Autónoma de Buenos Aires, Argentina, email: maier@qo.fcen.uba.ar 
1983-1985; Rial \& Barbosa 1983-1985; Aschero \& Podestá 1986; Edwards et al. 2000; Wainwright et al. 2000, 2002, 2004; Boschín et al. 2002; Smith \& Clark 2004; Maier et al. 2007; Vázquez et al. 2008; Galván Josa et al. 2010; Darchuk et al. 2010).

Sin embargo, durante el análisis fisicoquímico de muestras arqueológicas se generan nuevas preguntas que abren perspectivas de trabajo interdisciplinario tanto para los análisis en laboratorio, como respecto al lugar de emplazamiento del sitio donde se desplegaron las imágenes documentadas. El trabajo conjunto entre los profesionales del área de las ciencias naturales y de las humanas requiere el intercambio constante de preguntas y respuestas ancladas en puntos de vista distintos sobre el mismo objeto. De esta manera es posible generar observaciones que puedan describirse de forma objetiva, como así también que la información obtenida pueda ser comunicada en los ámbitos propios de cada disciplina.

El conocimiento del ambiente de emplazamiento de un sitio es de gran aporte a este tipo de investigaciones, ya que los materiales que componen las imágenes y los que se encuentran en su entorno están en constante interacción. El ambiente, concebido como la interacción permanente entre los componentes del medio natural y sociocultural, contiene muchos de los factores que causan alteraciones y pueden conducir al deterioro de las pinturas. $\mathrm{Al}$ respecto, los naturales se relacionan con las condiciones climáticas, geológicas, atmosféricas y ecológicas a las que está expuesto el sitio; mientras que los culturales o antrópicos, con las actividades como el turismo, el vandalismo o con la influencia de un ambiente modificado a partir de actividades industriales. Asimismo, las evidencias de alteraciones sobre una imagen o panel pueden ser macroscópicas o microscópicas. Las primeras se observan directamente en el momento de la toma de la muestra; mientras que las segundas son resultado de la aplicación en laboratorio o in situ de técnicas analíticas específicas (Smith et al. 1999; Pérez Alonso et al. 2004; Doménech-Carbó et al. 2009). A partir de ambas observaciones es posible plantear hipótesis sobre los procesos de alteración en las pinturas del sitio.

Sobre la base de lo expuesto consideramos que la conservación de las imágenes demanda un estudio fisicoquímico que considere tanto la identificación de los materiales que las componen, como el estudio de los procesos de alteración que determinan el estado de preservación de las mismas.

\section{ALTERACIONES EN LAS MANIFESTACIONES RUPESTRES}

La alteración y el posterior deterioro de sitios de arte rupestre involucran una gran variedad de factores. Entre los naturales se encuentran los factores climáticos, los gases atmosféricos, la composición de la roca, las condiciones ambientales a las que está expuesto el sitio (humedad, sol, temperatura, viento) y los biológicos (líquenes,

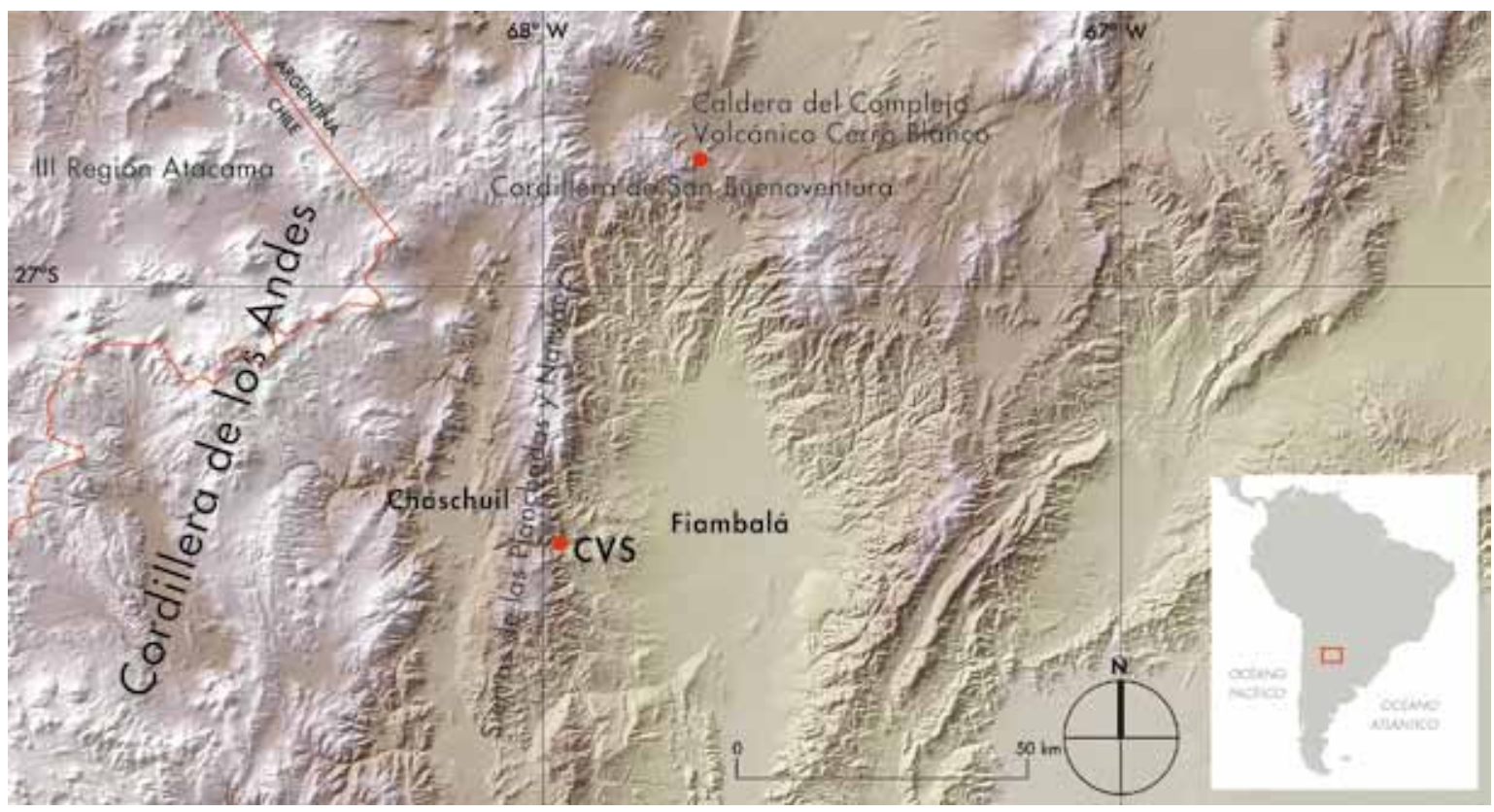

Figura 1. Ubicación de la cueva La Salamanca y referencias principales del oeste tinogasteño mencionadas en el texto. Figure 1. Location of La Salamanca cave and main points of reference in Western Tinogasta mentioned in the text. 
microorganismos, animales o vegetales). Por su parte, los factores antrópicos refieren al vandalismo (grafitis, roturas, extracciones), la actividad turística (cambios en la humedad del ambiente, fuego intencional, desgaste por roces, entre otros) y las actividades humanas con influencia en el ambiente, como la industrial (lluvia ácida y generación de gases de polución en el aire) y las obras de infraestructura (modificación de cursos de ríos, voladuras, entre otras) (Bednarik 1995, 2003; Wainwright 1995). De lo expuesto surge que el deterioro es un proceso que se genera a partir de la acción combinada de diversos factores, requiriendo para su identificación del trabajo en conjunto de las distintas disciplinas involucradas. Las evidencias de la acción de estos factores sobre las imágenes y los soportes rupestres pueden ser, como se mencionó anteriormente, macroscópicas o microscópicas. En relación con las macroscópicas, es muy importante la documentación de irregularidades en el momento del relevamiento cuando se describen el sitio y la muestra, por ejemplo, si hay filtraciones de agua, humedad, exposición al sol o al viento, si se observa el crecimiento de plantas o líquenes, y si hay evidencias de presencia de animales (nidos, deposiciones, entre otras). Microscópicamente, los procesos químicos producen cambios de coloración en zonas específicas, formaciones superficiales, acreciones o depósitos de sales y descamaciones. Estas alteraciones muchas veces se manifiestan en forma macroscópica evidenciándose directamente en el momento de la toma de la muestra; mientras que en otros casos su presencia se infiere a partir de la interpretación de los análisis fisicoquímicos.

\section{EL CASO DE LA CUEVA LA SALAMANCA}

La Salamanca (3385 msnm) es una cueva emplazada en formación granítica en ambiente precordillerano, específicamente en la vertiente oriental de las sierras de Las Planchadas y Narváez (departamento Tinogasta, Catamarca). ${ }^{1}$ Esta formación montañosa es la divisoria natural y conectora entre el valle mesotérmico de Fiambalá (1900 msnm) y la puna transicional de Chaschuil (3500 msnm) (figs. 1, 2). Su techo y laterales superiores presentan 60 representaciones pintadas en color rojo, distribuidas en 11 paneles delimitados por fisuras o irregularidades del soporte, que definen un espacio plástico total de $313,85 \mathrm{~m}^{2}$ (Tabla 1 y fig. 3). Los laterales bajos no fueron utilizados aunque estuvieron disponibles para su intervención, como así tampoco los de los sectores más internos de la cueva. El contexto general indica que, probablemente, solo se marcaron los lugares que recibían mejor iluminación natural.

Durante el relevamiento se tomaron muestras pigmentarias en diversas imágenes de cinco de los paneles y se realizó una excavación de cuatro $\mathrm{m}^{2}$ debajo del área de localización de las pinturas que no arrojó ningún tipo de evidencia material (Basile \& Ratto 2009, 2011).

La cueva está localizada en una quebrada estrecha de acceso restringido y elevada $20 \mathrm{~m}$ respecto del nivel del cauce del río Pie de la Cuesta por el que se ingresa. Su interior no recibe luz solar en forma directa, ya que la boca orienta al sur mientras que el talud-alero, al oeste. En conjunto conforman un lugar frío y húmedo, lo que se ve sustentado por la ausencia del registro de
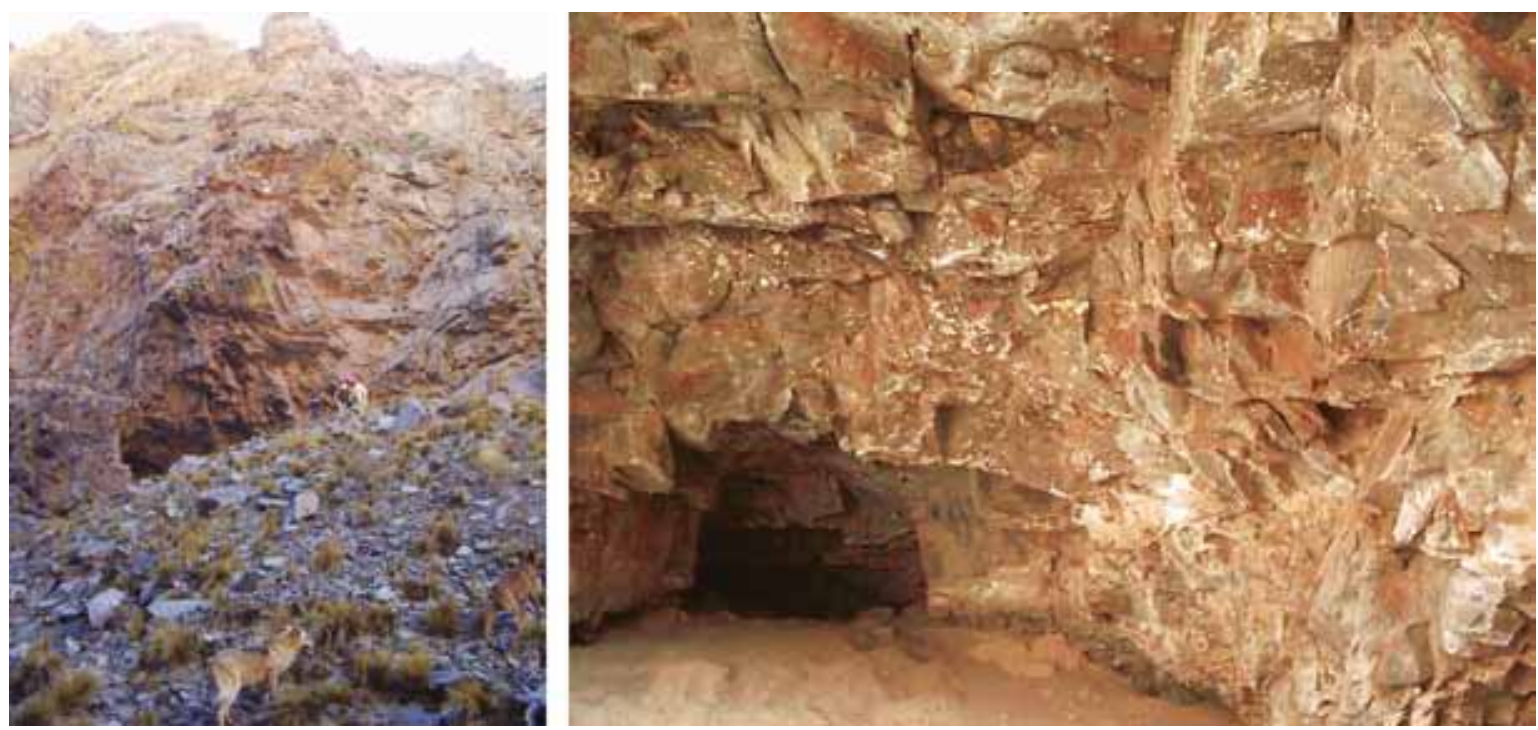

Figura 2. a) Vista del lugar de emplazamiento de la cueva La Salamanca; b) Detalle de las pinturas observándose al fondo la segunda abertura (ver fig. 3).

Figure 2. a) View of La Salamanca cave site; b) Detail of paintings, with second opening in the background (see fig. 3). 
Tabla 1. Descripción de los paneles y las representaciones registradas en la cueva La Salamanca. Referencias:

(*) Identificación de los motivos de donde provienen las muestras pigmentarias analizadas en este trabajo. Table 1. Description of rock art panels and representations recorded in La Salamanca cave. References: (*) Indicate the motifs from which the pigment samples analyzed herein were taken.

\begin{tabular}{|c|c|c|c|c|c|c|}
\hline \multicolumn{5}{|c|}{ PANELES } & \multicolumn{2}{|r|}{ REPRESENTACIONES } \\
\hline \multirow[b]{2}{*}{$\mathbf{N}^{\circ}$} & \multirow{2}{*}{ 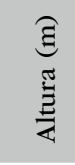 } & \multicolumn{2}{|c|}{ Distancia (m) } & \multirow[b]{2}{*}{ Ubicación } & \multirow{2}{*}{ ن } & \multirow[b]{2}{*}{ Descripción } \\
\hline & & $\begin{array}{l}1^{\text {a }} \text { Abertura } \\
(\mathrm{m})\end{array}$ & $\begin{array}{c}\text { Eje } \\
\text { oeste/ } \\
\text { este }\end{array}$ & & & \\
\hline \multirow{2}{*}{1} & \multirow{2}{*}{3,35} & \multirow{2}{*}{$-0,2$} & \multirow{2}{*}{0,08} & \multirow{2}{*}{$\begin{array}{l}\text { Techo del } \\
\text { lateral oeste }\end{array}$} & 1 & Cruces de contorno curvilíneo \\
\hline & & & & & 3 & Trazos rectilíneos y/o curvilíneos simples aislados \\
\hline \multirow{3}{*}{2} & \multirow{3}{*}{2,6} & \multirow{3}{*}{0,8} & \multirow{3}{*}{0,35} & \multirow{3}{*}{ Lateral oeste } & 1 & Cruces de contorno curvilíneo \\
\hline & & & & & 2 & Trazos rectilíneos y/o curvilíneos simples aislados \\
\hline & & & & & 2 & Zigzag en reflexión axial \\
\hline \multirow{4}{*}{3} & \multirow{4}{*}{2,35} & \multirow{4}{*}{1,9} & \multirow{4}{*}{1,1} & \multirow{4}{*}{$\begin{array}{l}\text { Techo del } \\
\text { centro-oeste }\end{array}$} & 1 & $\begin{array}{l}\text { Círculo o circunferencia simple, con punto o elementos } \\
\text { interiores }\end{array}$ \\
\hline & & & & & 1 & Trazados combinados rectilíneos-curvilíneos irregulares \\
\hline & & & & & 9 & Trazos rectilíneos y/o curvilíneos simples aislados \\
\hline & & & & & 1 & Zigzag en reflexión axial \\
\hline \multirow[t]{2}{*}{4} & \multirow[t]{2}{*}{1,8} & \multirow[t]{2}{*}{2,25} & \multirow[t]{2}{*}{1,15} & \multirow{2}{*}{$\begin{array}{l}\text { Techo centro/ } \\
\text { Centro-oeste }\end{array}$} & 1 & $\begin{array}{l}\text { Círculo o circunferencia simple, con punto o elementos } \\
\text { interiores }\end{array}$ \\
\hline & & & & & 2 & Zigzag en reflexión axial \\
\hline \multirow{3}{*}{5} & \multirow{3}{*}{2,1} & & & & 1 & Trazados combinados rectilíneos-curvilíneos irregulares ${ }^{(*)}$ \\
\hline & & 1,7 & 2,33 & Techo/Lateral & 7 & Trazos rectilíneos y/o curvilíneos simples aislados \\
\hline & & & & & 1 & Trazos rectilíneos y/o curvilíneos agrupados \\
\hline 6 & 2,2 & 0,9 & 3,10 & Lateral este & 1 & Trazados combinados rectilíneos-curvilíneos irregulares ${ }^{(*)}$ \\
\hline & & & & & 4 & Trazados combinados rectilíneos-curvilíneos irregulares \\
\hline & & & & & 1 & Cruces de contorno curvilíneo \\
\hline & & & & & 1 & Meandros de trazado simple o doble, abiertos y/o cerrados \\
\hline 7 & 2,7 & 0,35 & 2,83 & Techo & 2 & Puntos aislados, puntiformes alineados \\
\hline & & & & & 1 & Trazos rectilíneos y/o curvilíneos simples aislados \\
\hline & & & & & 1 & $\begin{array}{c}\text { Circunferencia con apéndice(s) inferior(es) simple o } \\
\text { concéntrica }\end{array}$ \\
\hline 8 & 2,21 & 0 & 3,65 & $\begin{array}{l}\text { Lateral este } \\
\text { en resalte }\end{array}$ & 1 & $\begin{array}{l}\text { Círculo o circunferencia simple, con punto o elementos } \\
\text { interiores }\end{array}$ \\
\hline & & & & & 5 & Trazos rectilíneos y/o curvilíneos simples aislados \\
\hline 9 & 2,3 & $-0,15$ & 3,6 & Techo & 1 & $\begin{array}{l}\text { Rectangulares o subrectangulares enmarcados con o sin } \\
\text { elementos interiores (cartuchos) }\end{array}$ \\
\hline & & & & & 2 & Trazos rectilíneos y/o curvilíneos simples aislados \\
\hline 10 & 2,45 & $-0,1$ & 3,30 & $\begin{array}{l}\text { Lateral este } \\
\text { en resalte }\end{array}$ & 1 & Trazados combinados rectilíneos-curvilíneos irregulares \\
\hline 11 & 2,6 & $-0,9$ & 3,32 & Lateral este & 3 & $\begin{array}{l}\text { Círculo o circunferencia simple, con punto o elementos } \\
\text { interiores }\end{array}$ \\
\hline & & & & & 3 & Trazos rectilíneos y/o curvilíneos simples aislados \\
\hline & & Total de repr & sentacior & & 60 & \\
\hline
\end{tabular}



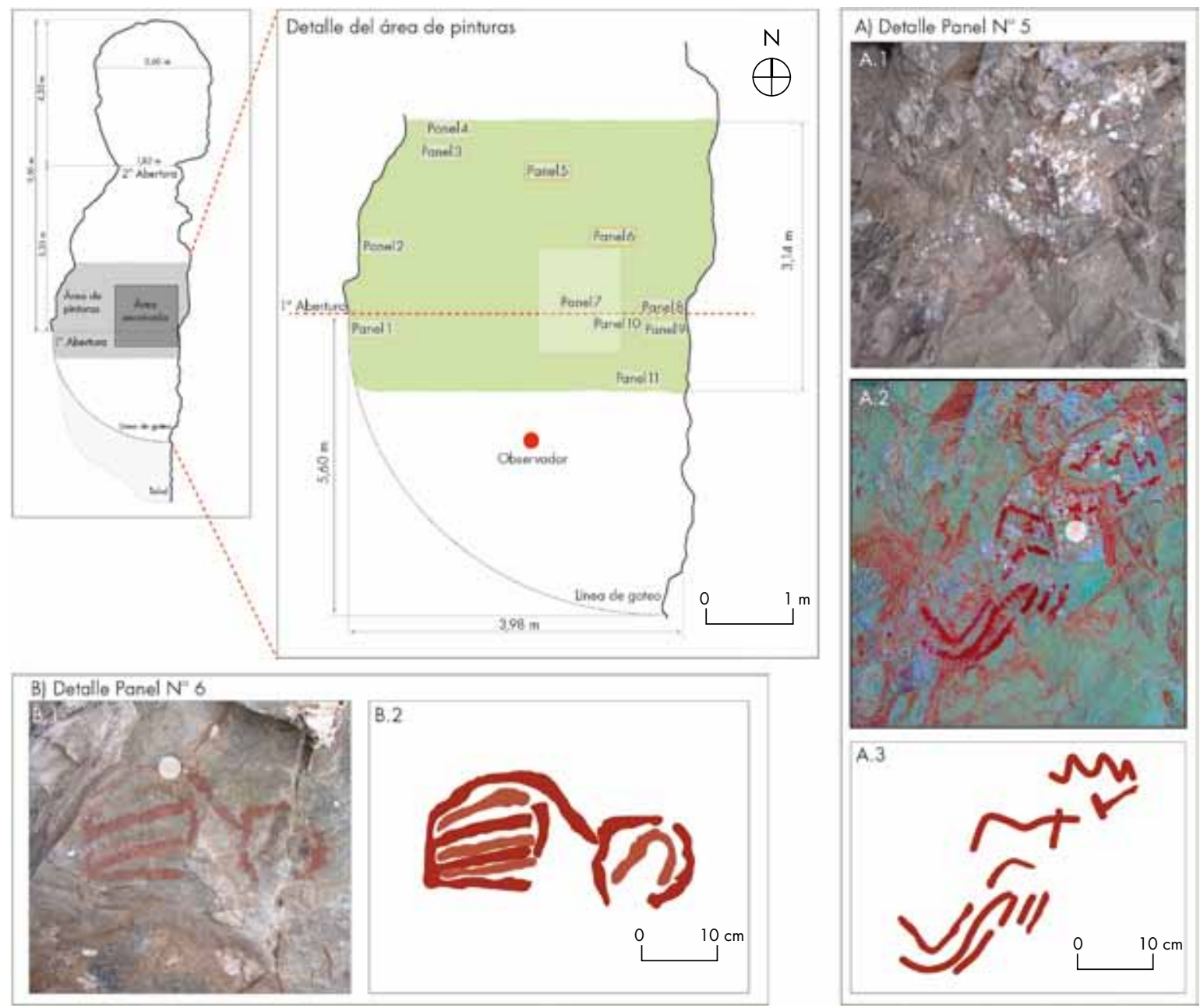

Figura 3. Planta del área de ubicación de los paneles y pinturas de la cueva La Salamanca. Detalle de los paneles de procedencia y del sector de toma de las muestras analizadas. La fotografía A.2 fue procesada digitalmente con el programa D-Stretch-Image J (Harman 2008 [2005]). Figure 3. Layout of the panels and paintings of La Salamanca cave. Detail of source panels and sampling area. Photograph A.2 was digitally processed using the D-Stretch-Image J program (Harman 2008 [2005]).

artefactos, ecofactos y rasgos en la excavación realizada; tampoco se han documentado manchas de hollín adheridas a las paredes rocosas (Basile \& Ratto 2011).

Las imágenes relevadas son exclusivamente nofigurativas, ya que no fue posible reconocer en ellas elementos totales o parciales que remitieran a algún referente en el mundo natural, que pudiera haber funcionado como modelo, sin que esto implique suponer la intención de sus autores de realizar una copia mimética. Por el contrario, consideramos que los límites precisos entre la figuración y la no figuración son muy difíciles de establecer y, en consecuencia, esta distinción reside simplemente en que -debido a la distancia semántica y temporal de nuestro análisis respecto del contexto cultural de producción de estas imágenes- no pudimos detectar unidades que nos permitieran remitir ciertos diseños a algún modelo conocido por nosotros (Aschero 1975). Entre ellas destacan los trazos lineales, las figuras circulares, las figuras en zigzag, los puntos y las cruces de contorno curvilíneo (Basile \& Ratto 2011).

No se registraron evidencias de superposiciones, mantenimiento o reciclado entre las representaciones, ni discrepancias en las tonalidades de las pátinas que pudieran indicar eventos de intervención visual diferenciales (Basile \& Ratto 2011). Tampoco fue posible datar por Espectrometría de Acelerador de Masa (AMS - Accelerator Mass Spectrometry) una muestra de pintura desprendida del panel $\mathrm{N}^{\circ} 2$ (Laboratorio University of Arizona, muestra CVS-21), ya que no contenía materia orgánica en cantidad suficiente. Así, la posibilidad de asignación cronológica de estas representaciones se restringe al establecimiento de similitudes de diseño 
entre ellas y las registradas en: (i) otros soportes materiales procedentes del área de investigación regional cuyos contextos cuenten con fechados radiocarbónicos, o (ii) los soportes rupestres de la vecina región de Antofagasta de la Sierra (ANS) que posee la ventaja de contar con una secuencia cronológica calibrada de más de diez mil años. $\mathrm{Al}$ respecto, solo algunas de las imágenes relevadas brindan elementos diagnósticos que permiten postular su asignación cronológica relativa (Basile \& Ratto 2011). Nos referimos específicamente a los rectángulos de contornos lineales cerrados que presentan altas similitudes con las figuras que Aschero y colaboradores (2006) denominaron "cartuchos" y que las ubican cronológicamente entre los años $500 \mathrm{AC}$ y 500 DC. Estas imágenes han sido registradas en asociación con caminos, espacios productivos, residenciales o funerarios e interpretadas como marcadores espaciales legitimadores de derechos de acceso a ciertos lugares o como elementos de protección necesarios tanto para el viaje en vida como en la muerte. El resto de las imágenes documentadas en La Salamanca no tiene antecedentes dentro de los repertorios regionales conocidos (Basile 2011) y se correlaciona con las modalidades estilísticas más antiguas definidas por Aschero (1999, 2006, 2013) para ANS. Estas particularidades, junto con la llamativa ausencia de fragmentos cerámicos durante las intervenciones realizadas, nos permiten plantear, en forma preliminar, que los diseños pintados de La Salamanca podrían remitir a tiempos del Arcaico final o inicios de las primeras sociedades agropastoriles del noroeste catamarqueño ( $c a .1000$ AC). Sin embargo, cabe aclarar que si bien las representaciones rupestres tienen la peculiaridad de ser potencialmente "aditivas" (Aschero 1996), es decir, que pueden ser recicladas o incorporadas a nuevos conjuntos en los que se suman nuevas imágenes, en este caso puntual se observa una discontinuidad en la marca. Esto se argumenta basándose en que no existen evidencias de imágenes que puedan correlacionarse con los repertorios regionales y extrarregionales esperables para momentos posteriores al año 1250 DC (Aschero 1996; Ratto et al 2000-2002; Basile 2011).

\section{MUESTRAS Y TÉCNICAS APLICADAS}

Para el análisis fisicoquímico se tomaron 12 muestras pigmentarias de cinco de los 11 paneles en los que se disponen las representaciones, además de una muestra del soporte rocoso sin pintura. Las muestras se extrajeron con un escalpelo de carburo de silicio tratando de provocar el menor impacto posible. Para ello se privilegiaron aquellos lugares que presentaban procesos de desprendimiento o descascaramiento natural. Las muestras se envolvieron en papel celofán y se conservaron en envases plásticos. Todas las muestras fueron analizadas por microscopia de barrido electrónico con detección de rayos X (SEM-EDS) observándose composiciones similares para todas las muestras. En este trabajo se presentan los resultados de dos de las muestras de las zonas pigmentadas de color rojo de los paneles $\mathrm{N}^{\circ} 5 \mathrm{y}$ $\mathrm{N}^{\circ} 6$ localizados en el techo y el lateral este de la cueva, respectivamente (Tabla 1 y fig. 3). Durante el relevamiento se realizó una descripción de las características del sitio y de su entorno y se documentaron y registraron los depósitos de sales en la superficie, así como de otras formaciones cristalinas. El primer objetivo del análisis consistió en determinar la composición del pigmento rojo para luego caracterizar las formaciones cristalinas.

La muestra del panel $\mathrm{N}^{\circ} 5$ fue analizada directamente sin tratamiento previo, mientras que la del 우 6 se incluyó en una resina acrílica y se pulió hasta obtener en forma nítida su secuencia estratigráfica. Las muestras fueron observadas bajo un estereomicroscopio Leica MZ6 y registradas con una cámara digital Canon S50. Se usó, además, un microscopio Carl Zeiss Axio Imager Z2m equipado con fuentes de luz visible y ultravioleta en modos normal y polarizado. Las imágenes fueron tomadas con una cámara AxioCam HRc usando el programa AxioVision para su adquisición y proceso.

La morfología de la superficie de las muestras se registró con un microscopio electrónico de barrido de campo ambiental (FE-SEM) Zeiss: Supra 40 acoplado con un microanalizador por espectroscopia de dispersión de energía (SEM-EDS) INCA X Sight, Oxford Instrument para el análisis elemental. Los espectros infrarrojos fueron obtenidos en un espectrómetro con transformada de Fourier (FTIR) Nicolet Magna 550. La medición por transmitancia de la muestra involucró la preparación de una pastilla con $\mathrm{KBr}$ (bromuro de potasio) y los espectros fueron registrados usando el programa Omnic 7.3 (Thermo Electron Corporation). Las medidas de espectroscopia Raman se llevaron a cabo en un equipo Renishaw System 1000 acoplado con un microscopio Leica DM LM del Museo de Arte Metropolitano de Nueva York.

\section{RESULTADOS DEL ANÁLISIS FISICOQUIIMICO}

La muestra del panel $N^{\circ} 6$ es de color rojo 10R 4/4 en la escala de Munsell (2000), conformando una partícula que también incorpora una parte del soporte rocoso (fig. 4a). Una imagen de microscopia óptica con un 

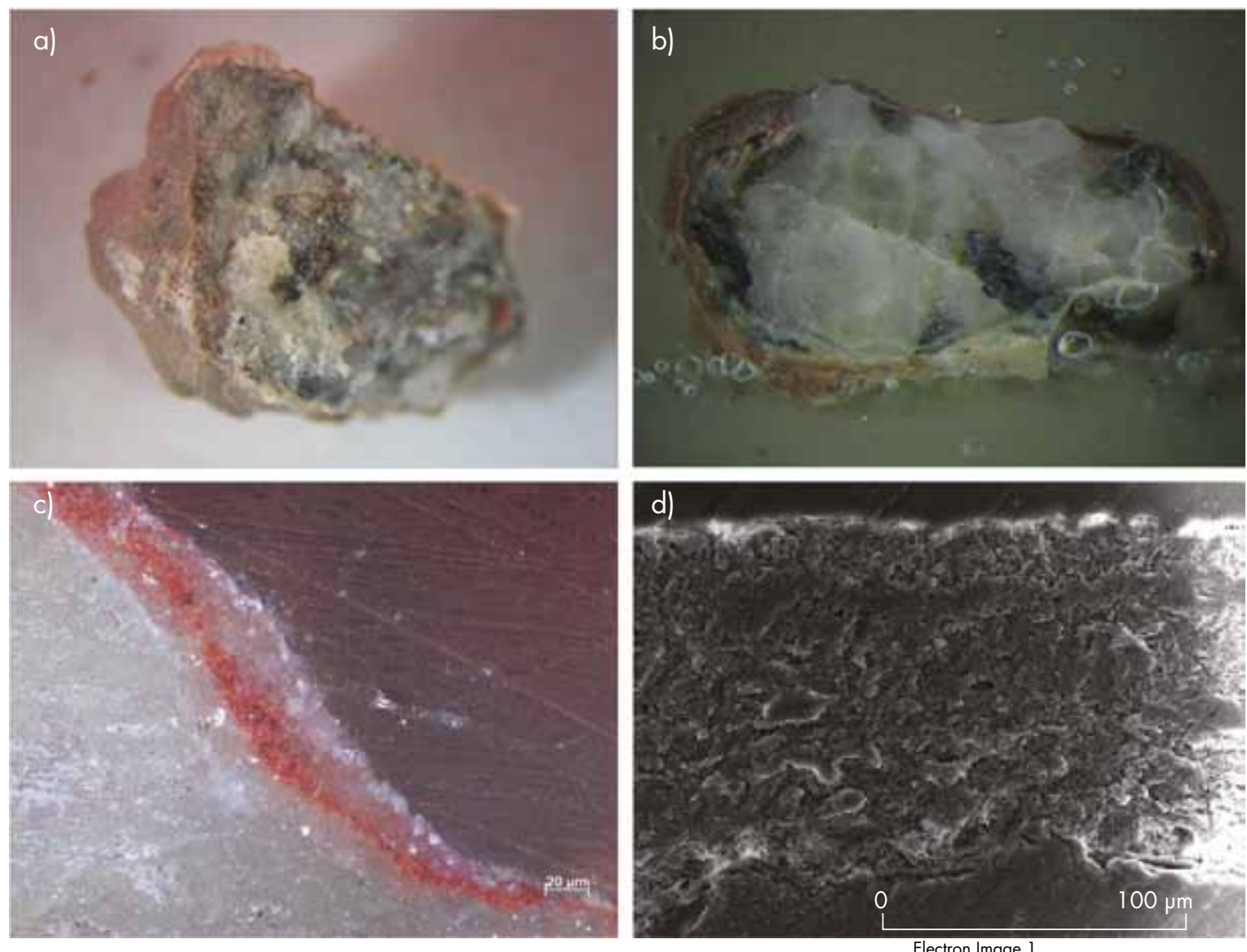

Figura 4. Muestra del panel $\mathrm{N}^{\circ}$ 6. a) Imagen de la muestra con microscopio estereoscópico; b) Imagen de la estratigrafía con microscopio estereoscópico; c) Imagen de la zona pigmentada de la estratigrafía de la muestra con microscopio óptico; d) Imagen de la zona pigmentada de la estratigrafía de la muestra con microscopio electrónico de barrido.

Figure 4. Sample from panel 6. a) Image of the sample under a stereoscopic microscope; b) Stratigraphic image under a stereoscopic microscope; c) Image of the pigment zone of the sample's stratigraphy under optical microscope; d) Image of the pigment zone of the sample's stratigraphy under scanning electron microscope.

aumento de 50 X mostró que la estratigrafía presentaba tres capas visibles, ubicándose la de coloración rojiza en la zona intermedia (figs. 4b, c). El análisis por SEM determinó la existencia de una morfología diferente para cada capa (fig. 4d). El análisis elemental mediante espectroscopia de dispersión de energía (EDS) reveló la presencia de hierro en la capa roja, mientras que las zonas blancas mostraron la presencia de calcio y azufre, correspondiente a yeso $\left(\mathrm{CaSO}_{4} \cdot 2 \mathrm{H}_{2} \mathrm{O}\right)$. El hierro podría formar parte de una especie cromófora como la hematita $\left(\mathrm{Fe}_{2} \mathrm{O}_{3}\right)$, compuesto que en baja cantidad puede conferir un intenso color rojo (Legodi \& De Waal 2007). El análisis por espectroscopia Raman confirmó la presencia de hematita como pigmento rojo y de yeso en cantidad apreciable en las capas blancas (fig. 5). Además, el análisis petrográfico de la roca soporte mostró la presencia de compuestos graníticos como componentes principales y la ausencia de yeso en su composición.

Por su parte, la muestra del panel $N^{\circ} 5$ es una escama de la superficie pigmentada que también presenta un color rojo 10R 4/4 de la escala Munsell (2000). En las imágenes del microscopio óptico y electrónico (SEM) se visualizaron claramente las heterogeneidades de la muestra (fig. 6). El análisis elemental, al igual que en el caso anterior, mostró la presencia de calcio y azufre en una relación uno a uno característica del yeso. El análisis también mostró la presencia de silicio, aluminio y metales alcalinos que sugieren la presencia de aluminosilicatos, posiblemente feldespatos de potasio (K) (ortoclasas), magnesio (Mg) y sodio (Na) (plagioclasas) en coincidencia con los resultados del análisis petrográfico de la roca soporte. Si bien el porcentaje 


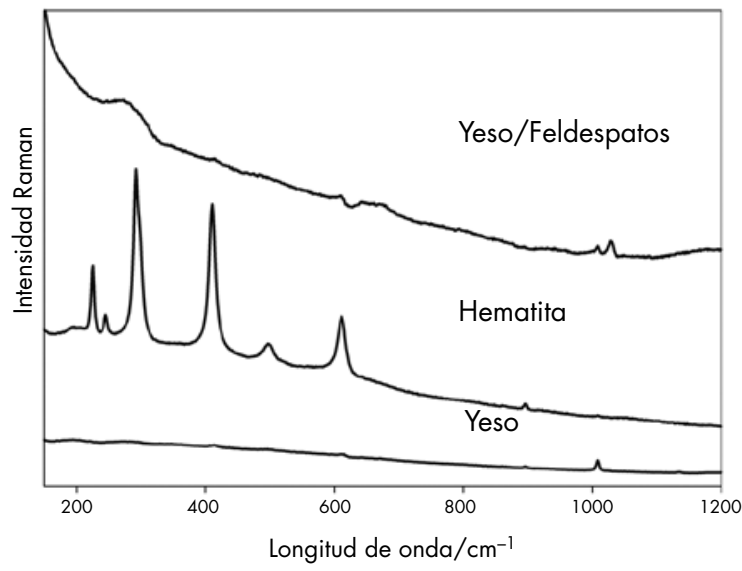

Figura 5. Muestra del panel $\mathrm{N}^{\circ}$ 6. Espectro Raman de las zonas interna blanca, intermedia roja y externa blanca (de arriba hacia abajo). Figure 5. Sample from panel 6. Raman spectrum of the interior black zone, middle red zone, and exterior white zone (from top to bottom).

atómico de hierro es bajo, puede corresponder también a la presencia de hematita responsable del color rojo de las imágenes plasmadas. Parte de la muestra fue pulverizada y analizada por espectroscopia infrarroja en modo transmitancia (FTIR) mostrando la presencia de las bandas de yeso como componente mayoritario (fig. 7) (Van der Marel \& Beutelspacher 1976; Anbalagan et al. 2009) y bandas de carbonato de calcio (calcita) y weddellita, una forma hidratada de oxalato de calcio (Frost 2004; Hernanz et al. 2006). Dado que las bandas que corresponden a yeso son muy intensas fue difícil distinguir las correspondientes a hematita y a los aluminosilicatos (Van der Marel \& Beutelspacher 1976). El espectro Raman (fig. 8) confirmó la presencia de yeso y weddellita (Frost 2004) en concordancia con lo observado por FTIR.

\section{DISCUSIÓN}

A partir de los resultados del análisis fisicoquímico se generaron distintas hipótesis sobre la presencia de calcita, yeso y wedellita en las muestras analizadas. En ambas se determinó que el compuesto responsable del color rojo es la hematita, mezclada probablemente con calcita, carbonato de calcio, identificado por espectroscopia infrarroja. La presencia de calcita se asocia a su agregado intencional como carga en la preparación del pigmento (Chalmin et al. 2003; Garate et al. 2004). Dado que la calcita es un componente minoritario en las muestras, su concentración pudo haber disminuido a lo largo del tiempo, considerando que los carbonatos son sustancias básicas que pueden reaccionar con gases ácidos de la
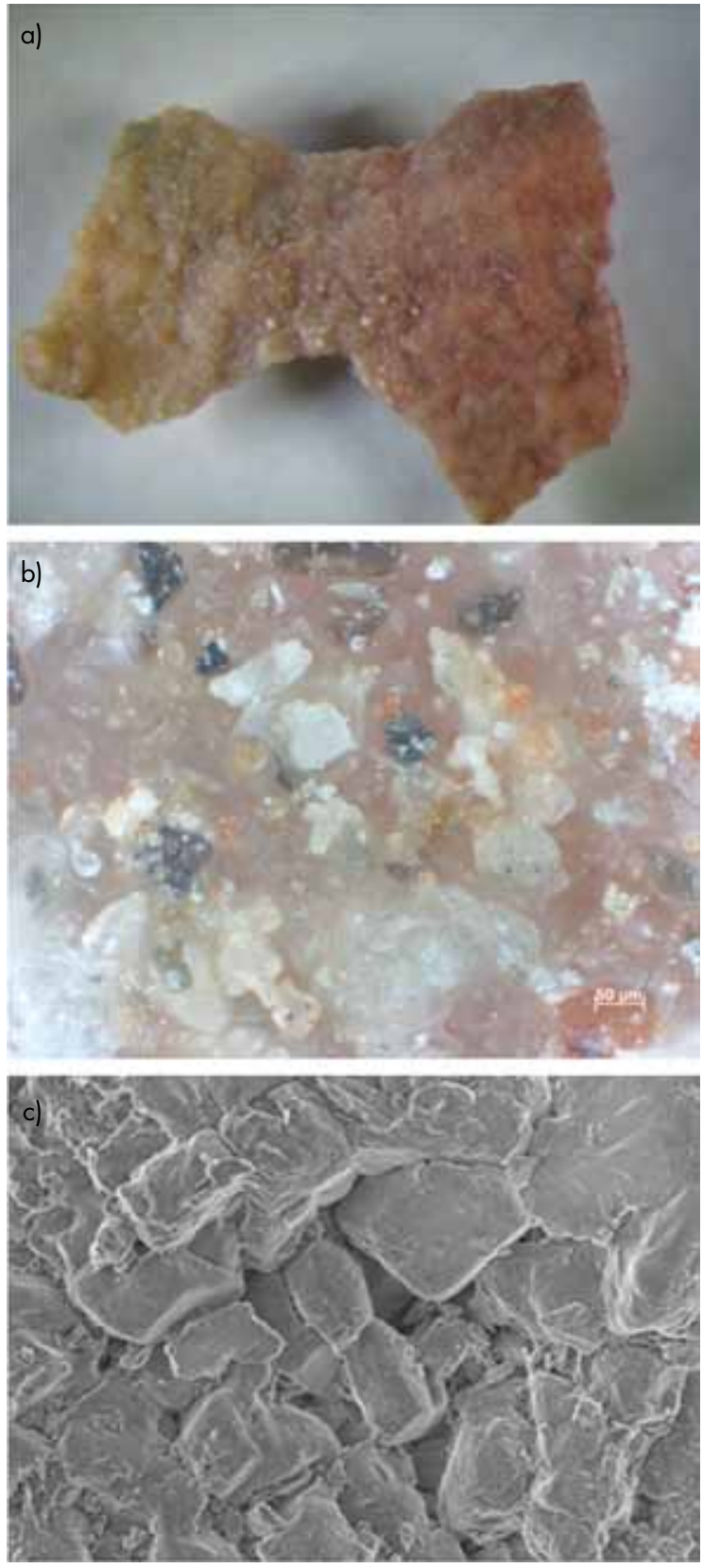

1 $\quad$ EmT $=3.00 \mathrm{kv} \quad W D=3.3 \mathrm{~mm} \quad \mathrm{Mag}=10.00 \mathrm{KX} \quad$ Signal $A=$ InLens

Figura 6. Muestra del panel $\mathrm{N}^{\circ} 5$. a) Imagen de la muestra con microscopio estereoscópico; b) Imagen de la muestra con microscopio estereoscópico; c) Imagen de la muestra con microscopio electrónico de barrido.

Figure 6. Sample from panel 5. a) Image of the sample under a stereoscopic microscope; b) Image of the sample under a stereoscopic microscope; c) Image of the sample under a scanning electron microscope.

atmósfera. Muchos trabajos postulan que la presencia de yeso $\left(\mathrm{CaSO}_{4} \cdot 2 \mathrm{H}_{2} \mathrm{O}\right)$ sobre las superficies es producto de la reacción ácido-base entre la calcita y el ácido sulfúrico $\left(\mathrm{H}_{2} \mathrm{SO}_{4}\right)$ generado a partir de la disolución de trióxido 


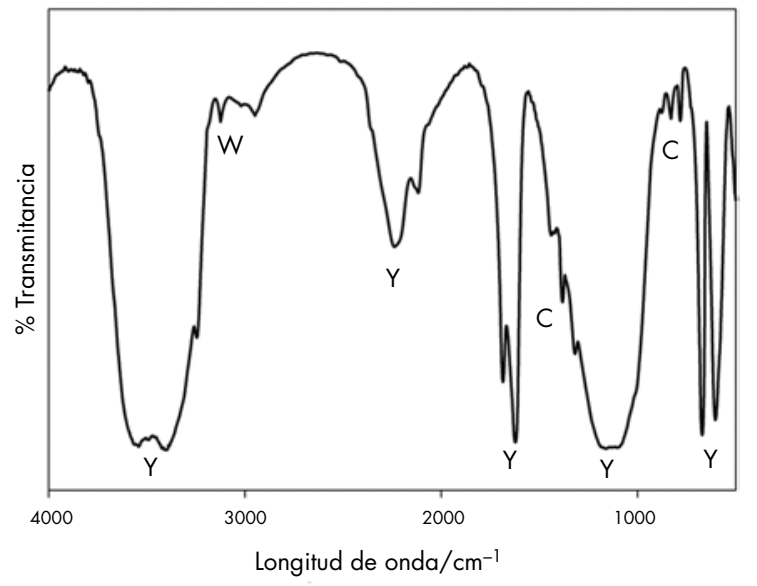

Figura 7. Muestra del panel $\mathrm{N}^{\circ} 5$. Espectro infrarrojo de la muestra. (Y: yeso, W: weddellita, C: calcita).

Figure 7. Sample from panel 5. Infrared spectrum of the sample. (Y: gypsum, W: weddellite, C: calcite).

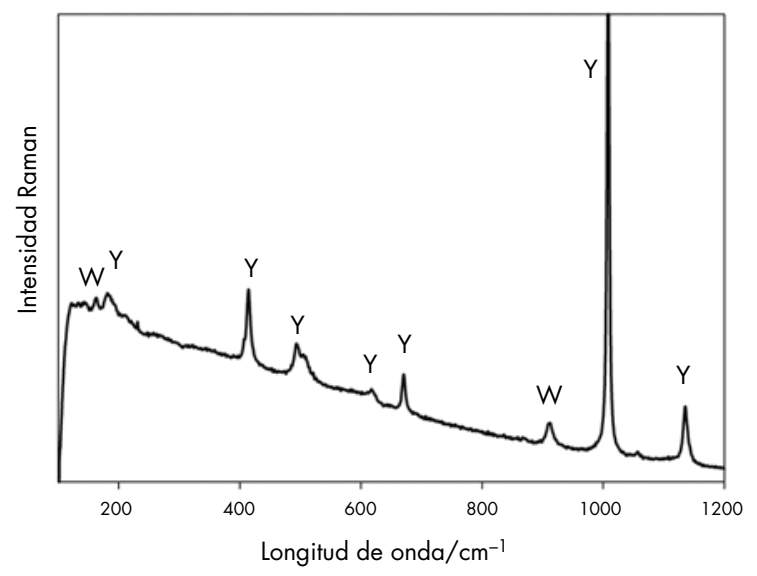

Figura 8. Muestra del panel $\mathrm{N}^{\circ}$ 5. Espectro Raman de la superficie de la muestra. (Y: yeso, W: weddellita).

Figure 8. Sample from panel 5. Raman spectrum of the surface of the sample. (Y: gypsum, W: weddellite).

de azufre $\left(\mathrm{SO}_{3}\right)$ gaseoso en agua o en un ambiente húmedo. En esta reacción, el carbonato se transforma en dióxido de carbono $\left(\mathrm{CO}_{2}\right)$ gaseoso que se libera a la atmósfera (Charola 2000, Charola \& Centeno 2002; Pérez Alonso et al. 2004).

De esta manera, el yeso reemplaza gradualmente al carbonato y migra desde la superficie exterior hacia la roca quedando el pigmento confinado entre dos capas de yeso que se ubican por encima y por debajo del material pigmentario (Charola 2000). La presencia de yeso en las muestras también podría ser el resultado de la incorporación intencional en la preparación de la mezcla pigmentaria o por infiltración del mineral a través de grietas, ya que el yeso está presente en las rocas sedimentarias del Paleozoico Superior y Cenozoico de la formación Las Planchadas (comunicación personal Dr. Fernando Hongn, febrero 2012). Sin embargo, la disposición estratigráfica del yeso, por arriba y por debajo de las pinturas, junto con su ausencia en la composición mineralógica de la roca soporte, permiten descartar tanto su origen geológico como su agregado intencional para la preparación de la mezcla pigmentaria.

Es factible suponer entonces que el ambiente que rodeaba a la cueva debió presentar niveles altos de óxidos de azufre gaseosos para que la reacción química se produjera. Dichos gases pueden provenir de la actividad industrial y/o de las emisiones de erupciones volcánicas (Amigo Ramos 2007). La actividad antrópica queda descartada dado que la cueva está emplazada en un lugar en el que no existen ni asentamientos rurales ni urbanos ni fábricas a menos de cien kilómetros de distancia.

Con respecto a la actividad volcánica es sugestivo que la cueva se emplaza al oriente del área conocida como "Los Seismiles" del oeste tinogasteño, donde se encuentran las montañas y los volcanes más altos de América en plena cordillera de los Andes (fig. 1). Asimismo, se encuentra a $70 \mathrm{~km}$ lineales en dirección sur-sudoeste de la cordillera de San Buenaventura donde se localizan las calderas del complejo volcánico Cerro Blanco, cuyo segundo evento ocurrió en una edad más joven que 5500 años AP (Montero et al. 2009, 2010; Ratto et al. 2012) (fig. 1). Por lo tanto, es posible sostener a modo hipotético que los niveles ricos en gases de dióxido de azufre provengan de actividad volcánica aunque se deberían realizar otro tipo de estudios, como por ejemplo de relaciones isotópicas de azufre (Thode 1991), para profundizar en este tema.

Además, el deterioro causado por la presencia de yeso se relaciona con los procesos de descamación, tal como se observa en las pinturas relevadas (Bednarik 2003; Mol \& Viles 2010).

En resumen, el yeso en las pinturas da cuenta de la acción de gases atmosféricos de origen natural volcánico que estuvieron presentes en el entorno de la cueva probablemente luego de haberse ejecutado las mismas. Este aspecto es muy interesante dado que este tipo de deterioro en regiones cercanas a zonas urbanas, que no es el caso de la cueva que nos ocupa, es causado por gases atmosféricos generados por la acción humana (Ausset et al. 1998).

Existe además otra evidencia clara de deterioro en las pinturas, pero producto de un factor biológico. La mayoría de los estudios sobre la formación de películas de oxalato de calcio sobre superficies de pinturas murales, monumentos y edificios coinciden en dar a este 
compuesto un origen metabólico de hongos y líquenes (Russ et al. 1999). El contenido de restos de weddellita (oxalato de calcio) sobre la superficie de las pinturas sugiere que estas han tenido contacto con líquenes cuyo crecimiento se favorece por la presencia de yeso (Ortega et al. 1994).

\section{CONCLUSIONES}

Las imágenes cuyas muestras aquí se analizan son trazos lineales, no figurativos, que no tienen antecedentes en los repertorios locales y cuyas características permitirían postular su correlación con las modalidades estilísticas más antiguas definidas por Aschero (2006, 2013) para la vecina región de Antofagasta de la Sierra.

Más allá de que la presencia de yeso pueda ser explicada a través de hipótesis alternativas, es posible afirmar que es producto de la historia ambiental de la cueva en la que se desplegaron las imágenes, descartándose que formara parte del agregado intencional para la preparación de las pinturas. Esto refuerza la idea de que los procesos químicos que alteran el aspecto o la composición de los materiales de una imagen dependen de las condiciones climáticas, geológicas, atmosféricas y ecológicas en las que se encuentra. Esto pone una luz de alerta hacia la interpretación directa de la presencia de yeso en pinturas rupestres como resultado de prácticas culturales dado que los resultados que aquí presentamos indican que, al menos en este caso, su registro es resultado de la historia ambiental de la cueva.

Consideramos también que la calcita identificada en una de las muestras habría sido mezclada con el compuesto responsable del color rojo (hematita) durante la preparación de los pigmentos.

Queda pendiente, como agenda de trabajo a futuro, analizar las muestras del resto de los paneles de esta cueva para determinar si las tendencias aquí registradas son constantes en todos ellos o si, por el contrario, hay variaciones interpaneles. Esto último tiene especial relevancia, ya que podría constituirse en un indicador temporal relativo de valor fundamental para la investigación arqueológica.

La potencialidad del trabajo interdisciplinario conduce a que surjan también nuevas interrogantes que demandan integrar en la discusión a otras disciplinas. Particularmente, cuestiones referidas a la relación entre la realización de las pinturas y el vulcanismo regional o la implicación de cambios climáticos vinculados con el registro de oxalato de calcio producto de la presencia de líquenes que no se observaron en el sitio en el momento de su relevamiento (Moore et al. 2000). Esta sal puede permanecer sobre la superficie de las pinturas aun cuando el organismo productor ya no esté presente.

Consideramos que el procedimiento de adquisición, documentación y registro de las muestras como así también la continua interacción entre los profesionales de las ciencias humanas y químicas constituyeron los pilares que permitieron identificar los síntomas de deterioro y generar hipótesis que dieran cuenta de su presencia. En este trabajo los análisis químicos y espectroscópicos se utilizaron no solo para determinar la composición de pigmentos y otros compuestos, sino también para conocer y comprender las transformaciones que ocurrieron, es decir, permitieron identificar los distintos factores que causaron el deterioro.

Finalmente, la preservación y conservación de objetos culturales requiere un conocimiento profundo tanto de los materiales con los que fueron manufacturados, como de los factores que causaron su deterioro. Esto revela la necesidad de generar un protocolo de trabajo cuyos lineamientos pauten e integren diferentes aspectos relevantes y significativos durante el relevamiento de las pinturas rupestres y en la elección y secuencia de aplicación de cada una de las técnicas analíticas a ser utilizadas. Esto redundará seguramente en la interpretación de los resultados obtenidos, permitiendo además responder preguntas que guíen las investigaciones de ambas disciplinas.

RECONOCIMIENTOS Las autoras agradecen a la Dra. Silvia Centeno, del Scientific Department of the Metropolitan Museum of Art of New York, cuyo aporte fue fundamental en el uso de la espectroscopia Raman; a la Dra. Sonia Quenardelle, del Departamento de Geología de la Facultad de Ciencias Exactas y Naturales de la Universidad de Buenos Aires; a Matthias Strecker, de la Sociedad de Investigación del Arte Rupestre de Bolivia (SIARB), por su colaboración en el mejoramiento digital de la fotografía del panel $\mathrm{N}^{\circ} 5$; a los coordinadores del simposio "Arqueometría de pigmentos" del IV Congreso Argentino de Arqueometría, M. A. López, G. de la Fuente y D. Fiore; al Consejo Nacional de Investigaciones Científicas y Técnicas (CONICET) y a la Agencia Nacional de Promoción Científica y Tecnológica (ANPCYT) por el financiamiento. Eugenia Tomasini y Mara Basile agradecen al CONICET por la beca posdoctoral; Marta Maier es Investigadora de CONICET. Los relevamientos arqueológicos se realizaron en el marco del PICT-2007-1539 y el UBACYT F-139.

\section{NOTAS}

${ }^{1}$ La Dra. Sonia Quenardelle (Cátedra Petrografía, Depto. de Cs. Geológicas, FCEN-UBA) realizó el análisis del corte petrográfico de una muestra del soporte rocoso de la cueva. Se trata de un granito con presencia de plagioclasa, ortosa, cuarzo, biotita, anfíbol, hornblenda, titanita o esfena (silicato de calcio y titanita), minerales opacos (óxidos de Fe), magnetita e ilmenita, clorita (mineral de alteración de la biotita), sericita y esmectita (alteración de plagioclasas). 


\section{REFERENCIAS}

Amigo Ramos, A., 2007. Volcán Lascar: Aporte de dispersión de azufre oxidado a la atmósfera regional. Memoria para optar el título de Geólogo, Departamento de Geología, Universidad de Chile.

Anbalagan, G.; S. Mukundakumari, K. Sakthi Murugesan \& S. GuNASEKARAN, 2009. Infrared, optical absorption and EPR spectroscopic studies on natural gypsum. Vibrational Spectroscopy 50: 226-230.

Aschero, C., 1975. Motivos y objetos decorados del sitio precerámico Inca Cueva 7 (Provincia de Jujuy). Antiquitas 20-21: 2-7, Buenos Aires.

- 1983-1985. Pinturas rupestres en asentamientos cazadoresrecolectores. Dos casos de análisis aplicando difracción de rayos X. Cuadernos del Instituto Nacional de Antropología y Pensamiento Latinoamericano 10: 291-306, Buenos Aires.

— 1996. Arte y arqueología: Una visión desde la Puna argentina. Chungara 28 (1 y 2): 175-197.

- 1999. El arte rupestre del desierto puneño y el Noroeste Argentino. En Arte Rupestre en los Andes de Capricornio, J. Berenguer \& F. Gallardo, Eds., pp. 97-136. Santiago: Museo Chileno de Arte Precolombino.

- 2006. De cazadores y pastores. El arte rupestre de la modalidad río Punilla en Antofagasta de la Sierra y la cuestión de la complejidad en la Puna Meridional argentina. En Tramas en la piedra. Producción y usos del arte rupestre, D. Fiore \& M. M. Podestá, Eds., pp. 103-140. Asociación Amigos del Instituto Nacional de Antropología (AINA), World Archaeological Congress (WAC) y Sociedad Argentina de Antropología. Buenos Aires: Altuna Impresores.

- 2013. Arte rupestre, contexto y sociedad en el desierto puneño. Tesis doctoral en preparación. Facultad de Filosofía y Letras, Universidad de Buenos Aires.

Aschero, C. \& M. M. Podestá, 1986. El arte rupestre en asentamientos precerámicos de la puna argentina. Runa XVI: 29-57, Buenos Aires.

Aschero, C.; A. Martel \& S. López Campeny, 2006. Tramas en la piedra: Rectángulos con diseños geométricos en Antofagasta de la sierra (puna meridional, Argentina). En Tramas en la piedra. Producción y usos del arte rupestre, D. Fiore \& M. M. Podestá, Eds., pp. 141-156. Asociación Amigos del Instituto Nacional de Antropología (AINA), World Archaeological Congress (WAC) y Sociedad Argentina de Antropología. Buenos Aires: Altuna Impresores.

Ausset, P.; F. Bannery, M. Del Monte \& R. A. Lefevre, 1998. Recording of pre-industrial atmospheric environment by ancient crusts on stone monuments. Atmospheric Environment 32 (16): 2859-2863.

Basile, M., 2011. Continuidades y rupturas en las representaciones plásticas del Formativo (ca. 200 AD) a la ocupación incaica (ca. 1480 AD) en la Región de Fiambalá (Catamarca). Tesis Doctoral inédita. Facultad de Filosofía y Letras, Universidad de Buenos Aires.

Basile, M. \& N. RATTO, 2009. Interacting images: Analysis of rock-art paintings and engravings in western Tinogasta from 2500 to 1300 BP (province of Catamarca, Argentina). Fundhamentos IX (4): 1269-1283. Piauí: Fundação Museu do Homen Americano. 2011. Imágenes sobre rocas del sudoeste tinogasteño (Catamarca, ca. 2500 y 1300 AP). Revista Arqueología 17: 13-34. Instituto de Arqueología, Facultad de Filosofía y Letras, Universidad de Buenos Aires.

BeDnARIK, R., 1995. Conservación del arte rupestre en Australia. Administración y conservación de sitios de arte rupestre: Contribuciones al estudio del arte rupestre sudamericano 4: 9-21. La Paz: Sociedad de Investigación del Arte Rupestre en Bolivia.

2003. Natural deterioration of rock art. Rock art conservation [online] <http://mc2.vicnet.net.au/home/conserv/web/natural. html> [Citado 07-04-10].

Boschín, M. T.; A. M. Seldes, M. S. Maier, R. M. Casamiquela, R. LEDESMA \& G. ABAD, 2002. Análisis de las fracciones inorgánica y orgánica de pinturas rupestres y pastas de sitios arqueológicos de la Patagonia Septentrional Argentina. Zephyrus 55: 183-198, Salamanca.

Chalmin, E.; M. Menu \& C. Vignaud, 2003. Analysis of rock art painting and technology of Palaeolithic painters. Measurement Science and Technology 14: 1590-1597. Londres: Institute of Physics Publishing

Charola, A. E., 2000. Salts in the Deterioration of Porous Materials: An Overview. Journal of the American Institute for Conservation 39 (3): 327-343.

Charola, A. E. \& S. A. Centeno, 2002. Analysis of Gypsum-Containing Lime Mortars: Possible Errors Due to the Use of Different Drying Conditions. Journal of the American Institute for Conservation 41 (3): 269-278.

Darchuk, L.; Z. Tsybrit, A. Worobiec, C. Vázquez, O. M. Palacios, E. A. Stefaniak, G. Gatto Rotondoa, F. Sizov \& R. Van Grieken, 2010. Argentinean prehistoric pigments' study by combined SEM/EDX and molecular spectroscopy. Spectrochimica Acta Part A 75: 1398-1402.

Doménech-Carbó, A.; M. T. Doménech-Carbó \& V. A. Costa, 2009. Application of Instrumental Methods in the Analysis of Historic, Artistic and Archaeological Objects in Electrochemical Methods in Archaeometry, Conservation and Restoration, Monographs in Electrochemistry Springer-Verlag Berlin Heidelberg.

Edwards, H. G. M.; E. M. Newton \& J. Russ. 2000. Raman spectroscopic analysis of pigment and substrata in prehistoric rock art. Molecular Structure 245: 550-551.

Frost, L., 2004. Raman spectra of natural oxalates. Analytical Chimica Acta 207-214.

Galván Josa, V.; S. R. Bertolino, A. Laguens, J. A. Riveros \& G. Castellano, 2010. X-ray and scanning electron microscopy archaeometric studies of pigments from the Aguada culture, Argentina. Microchemical Journal 96: 259-268.

Garate, D.; E. Laval \& M. MEnu, 2004. Etude de la matière colorante de la grotte d'Arenaza. L'anthropologie 108: 251-289, París.

Guineau, B.; M. Lorblanchet, B. Gratuze, L. Dulin, P. Roger, R. Akrich \& F. Muller, 2001. Manganese black pigments in prehistoric paintings: The case of the Black Frieze of Pech Merle (France). Archaeometry 43: 211-225.

Harman, J., 2008 [2005]. Using Decorrelation Stretch to Enhance Rock Art Images. <http://dstretch.com/AlgorithmDescription.html> [Citado 16-01-2013].

Hernanz, A.; J. M. Gavira-Vallejo \& J. F. Ruiz-López, 2006. Introduction to Raman microscopy of prehistoric rock paintings from the Sierra de las Cuerdas, Cuenca, Spain. Journal of Raman Spectroscopy 37: 1054-1062.

LEgodi, M. A. \& D. De WAAL, 2007. Raman spectroscopy study of ancient South African domestic clay pottery. Spectrochimica Acta A 66: 135-142.

Maier, M. S.; D. L. A. De Faria, M. T. Boschín, S. D. Parera, M. F. Del Castillo \& M. F. Bernal, 2007. Combined use of vibrational spectroscopy and GC-MS methods in the characterization of archaeological pastes from Patagonia. Vibrational Spectroscopy 44: 182-186.

Mol, L. \& H. A. VILES, 2010. Geoelectric investigations into sandstone moisture regimes: Implications for rock weathering and the deterioration of San Rock Art in the Golden Gate Reserve, South Africa. Geomorphology 118: 280-287.

Montero López, M. C.; F. Hongn, R. Seggiaro, R. Marret \& N. Ratto, 2009. Relación entre el volcanismo y los registros arqueológicos en el bolsón de Fiambalá (Departamento Tinogasta, Catamarca). En Entrelazando ciencias: Sociedad y ambiente antes de la conquista española, N. Ratto, Comp., pp. 131-158. Buenos Aires: Eudeba.

Montero López, M. C.; F. Hongn, J. A. Brod, R. Seggiaro, R. Marrett \& M. Sudo, 2010. Magmatismo ácido del Mioceno SuperiorCuaternario en el área de Cerro Blanco-La Hoyada, Puna Sur. Revista de la Asociación Geológica Argentina 67 (2): 327-346, Buenos Aires.

Moore, S.; M. J. Beazley, M. R. Mccallum \& J. Russ. 2000. Can calcium oxalate residues from lichen activity reflect past climate 
change? In Preprints of Extended Abstracts Presented at the 220th ACS National Meeting, Washington, DC, August 20-24, 2000, M. L. Trehy Ed., 4-5. Preprints of Extended Abstracts, Vol. 40, $\mathrm{N}^{\circ}$ 2. [United States]: American Chemical Society, Division of Environmental Chemistry.

MunSEll COLOr, 2000. Munsell Soil color charts. New Windsor, N. Y.: Gretag Macbeth.

Ortega, J. J.; X. Arino, L. J. Stal \& C. Saiz, 1994. Cyanobacterial Sulfate Accumulation from Black Crust of a Historic Building. Geomicrobiological Journal 12: 15-22, Philadelphia.

Pérez Alonso, M.; K. Castro, I. Martinez Arkarazo, M. Angulo, M. A. Olaż́BAL \& J. M. Madariaga, 2004. Analysis of bulk and inorganic degradation products of stones, mortars and wall paintings by portable Raman microprobe spectroscopy. Analytical and Bioanalytical Chemistry 379: 42-50. Nueva York: Springer.

Ratto, N.; M. Orgaz \& S. CALETTI, 2000-2002. Relevamiento arqueológico del campo de grabados de Guanchincito (Fiambalá, depto. Tinogasta, Catamarca). Cuadernos del Instituto Nacional de Antropología y Pensamiento Latinoamericano 19: 551-572, Buenos Aires.

Ratto, N.; C. Montero \& F. Hongn, 2012. Environmental instability in western Tinogasta (Catamarca) during middle Holocene and its relation to regional cultural development. Quaternary International [online] <http://dx.doi.org/10.1016/j. quaint.2012.09.014> [Citado 16-01-2013].

Rial, G. E. \& C. E. Barbosa, 1983-1985. Análisis mineralógico por difracción de rayos $\mathrm{X}$ de muestras de pinturas del sitio Inca Cueva 4 (departamento Humahuaca, provincia de Jujuy). Cuadernos del Instituto Nacional de Antropología y Pensamiento Latinoamericano 10: 313-317, Buenos Aires.

Russ, J.; W. D. Kaluarachchi, L. Drummond \& H. G. M. Edwards, 1999. The nature of a whewellite-rich rock crust associated with pictographs in southwestern Texas. Studies in Conservation 44: 91-103. Londres: International Institute for Conservation of Historic and Artistic Works.

Scott, D. A. \& W. D. Hyder, 1993. A study of some Californian Indian rock art pigments. Studies in conservation 38: 155-173, Londres: International Institute for Conservation of Historic and Artistic Works.

SMITH, D. C.; M. BOUCHARD \& M. LORDBLANCHET, 1999. An initial Raman microscopic investigation of prehistoric rock art in caves of the Quercy District, S. W., France. Journal of Raman Spectroscopy 30: $347-354$

Smith, G. D. \& R. J. H. Clark, 2004. Raman microscopy in archaeological science. Journal of Archaeological Science 31: 1137-1160.
THode, H. G., 1991. Suphur isotopes in nature and the environment: an overview. En Stable isotopes: Natural and anthropogenic sulphur in the environment, H. R. Krouse \& V. A. Grinenko, Eds., pp. 1-26. Chichester: John Wiley \& Sons.

Van Der Marel, H. W. \& H. Beutelspacher, 1976. Atlas of infrared spectroscopy of clay minerals and their admixtures. Amsterdam / Oxford / New York: Elsevier Scientific Publishing Company.

Vázquez, C.; M. S. Maier, S. D. Parera, H. Yacobaccio \& P. Solá, 2008. Combining TXRF, FT-IR and GC-MS information for identification of inorganic and organic components in black pigments of rock art from Alero Hornillos 2 (Jujuy, Argentina). Analytical and Bioanalytical Chemistry 391: 1381-1387. Nueva York: Springer.

Vignaud, C.; H. Salomon, E. Chalminc, J. M. Genested \& M. Menu, 2006. Le groupe des "bisons adossés" de Lascaux. Étude de la technique de l'artiste par analyse des pigments. L'anthropologie 110: 482-499, París.

Wainwright, I. N. M., 1995. Conservación y registro de pinturas rupestres y petroglifos en Canadá, Administración y conservación de sitios de arte rupestre, Contribuciones al estudio del arte rupestre sudamericano 4: 52-81. La Paz: Sociedad de Investigación del Arte Rupestre de Bolivia.

Wainwright, I. N. M.; K. Helwig, M. M. Podestá \& C. Belelli, 2000. Analysis of pigments from rock painting sites in Rio Negro and Chubut Provinces. En Arte en las rocas. Arte rupestre, menbires y piedras de colores en la Argentina, M. M. Podestá \& M.de Hoyos, Eds., pp. 203-206. Buenos Aires: Sociedad Argentina de Antropología y Asociación de Amigos del Instituto Nacional de Antropología y Pensamiento Latinoamericano.

Wainwright, I. N. M.; K. Helwig, D. S. Rolandi, C. Gradin, M. M. Podestá, M. ONetTo \& C. Aschero, 2002. Rock paintings conservation and pigment analysis at Cueva de las Manos and Cerro de los Indios, Santa Cruz (Patagonia), Argentina. En Preprints, 13th Triennial Meeting Rio de Janeiro, Vol. 2, ICOM Committee for Conservation, R. Vontobel, Ed., pp. 582-589 Londres: James and James.

Wainwright, I. N. M.; K. Helwig, D. S. Rolandi, C. Gradin, M. M. PODESTÁ \& M. ONETTO, 2004. Analysis of rock paintings pigments at Valle de las Pinturas (Lihue Calel National Parks) and the Chicalco sites La Pampa, Argentina. En La región pampeana. Su pasado arqueológico. Selección de trabajos presentados al I Congreso de Arqueología de la Región Pampeana Argentina (Venado Tuerto, Santa Fe, 1998), C. J. Gradin \& F. Oliva, Eds., pp. 417-421. Buenos Aires: Sociedad Argentina de Antropología, CEAR, Laborde Editor. 